\title{
Social aversive generalization learning sharpens the tuning of visuocortical neurons to facial identity cues
}

\author{
Yannik Stegmann ${ }^{1 *}$, Lea Ahrens ${ }^{1}$, Paul Pauli ${ }^{1,2}$, Andreas Keil ${ }^{3}$, Matthias J Wieser ${ }^{1,4}$ \\ 1'Department of Psychology (Biological Psychology, Clinical Psychology, and \\ Psychotherapy), University of Würzburg, Würzburg, Germany; ${ }^{2}$ Center for Mental \\ Health, Medical Faculty, University of Würzburg, Würzburg, Germany; ${ }^{3}$ Center for \\ the Study of Emotion and Attention, University of Florida, Gainesville, United \\ States; ${ }^{4}$ Department of Psychology, Education, and Child Studies, Erasmus \\ University Rotterdam, Rotterdam, Netherlands
}

\begin{abstract}
Defensive system activation promotes heightened perception of threat signals, and excessive attention to threat signals has been discussed as a contributory factor in the etiology of anxiety disorders. However, a mechanistic account of attentional modulation during fear-relevant processes, especially during fear generalization remains elusive. To test the hypothesis that social fear generalization prompts sharpened tuning in the visuocortical representation of social threat cues, 67 healthy participants underwent differential fear conditioning, followed by a generalization test in which participants viewed faces varying in similarity with the threat-associated face. We found that generalization of social threat sharpens visuocortical tuning of social threat cues, whereas ratings of fearfulness showed generalization, linearly decreasing with decreasing similarity to the threat-associated face. Moreover, individuals who reported greater anxiety in social situations also showed heightened sharpened tuning of visuocortical neurons to facial identity cues, indicating the behavioral relevance of visuocortical tuning during generalization learning.
\end{abstract}

*For correspondence: yannik.stegmann@uni-wuerzburg. de

Competing interests: The authors declare that no competing interests exist.

Funding: See page 15

Received: 16 January 2020

Accepted: 08 June 2020

Published: 09 June 2020

Reviewing editor: Jonas Obleser, University of Lübeck, Germany

(c) Copyright Stegmann et al. This article is distributed under the terms of the Creative Commons Attribution License, which permits unrestricted use and redistribution provided that the original author and source are credited.

\section{Introduction}

Selectively perceiving and differentially responding to cues associated with threat versus safety is a fundamental function of the vertebrate brain. The dysregulation of these functions is at the core of many psychiatric problems. Over the past decade, basic and applied researchers interested in mental health have focused on the contribution of dysfunctional associative learning mechanisms to the etiology of anxiety disorders (Dymond et al., 2015). Given its intuitive relation with exaggerated fear and anxiety, the process of overgeneralization-showing threat responses to safety cues that resemble threat-associated stimuli-has been of particular interest. However, previous clinical and translational work has yielded contradictory findings. While some authors observed overgeneralization in patients with anxiety disorders (Kaczkurkin et al., 2017; Lissek et al., 2014b; Lissek et al., 2010), others did not (Ahrens et alı, 2016; Tinoco-González et alı, 2015). This lack of convergent findings may be due to the fact that different physiological systems respond differently to varying similarity with a fear stimulus.

When individuals are in a state of fear, defensive mechanisms are activated with the goal of engaging in adaptive action, for example in fighting or escaping the threat. This defensive engagement is indexed by somato-visceral measures, such as fear-potentiated startle, skin conductance, and cardiovascular responses (Boecker and Pauli, 2019; Bradley and Lang, 2000). These measures have been considered in previous fear generalization experiments (Ahrens et al., 2016; Lissek et al., 2010; Torrents-Rodas et al., 2013), with mixed results regarding the nature and 
variability of fear generalization across a range of cues varying in similarity with a threat cue (Ahrens et al., 2016). In addition to preparing autonomic and motor efferent systems for action, defensive mobilization also includes heightened sensory processing, that is, perception and attention to threat (Robinson et al., 2013). In line with this notion, a substantial body of research has shown that stimuli predicting threat are attended more than neutral cues, and that heightened attention toward threatening stimuli is pronounced in patients with anxiety disorders (Bar-Haim et al., 2007). At the same time, several studies focused on the importance of perceptual discriminability of threatening stimuli (Struyf et al., 2017; Zaman et al., 2019). As a consequence, excessive attention to threatening stimuli has been discussed as causal or contributory in the etiology of anxiety disorders (Clark and Wells, 1995; Rapee and Heimberg, 1997). Direct neurophysiological evidence of heightened attention to threat in clinical disorders is scarce, however, and findings of both heightened and diminished attention have been reported. The absence of a mechanistic account and lack of direct unequivocal evidence of attention dysfunction may be a result of the using indirect measures of attention to threat. The present study uses electrophysiological measurements from visual cortex to test mechanistic hypotheses derived from the structure and function of the human visual system.

Direct visuocortical responses to a specific stimulus may be quantified with the steady-state visually evoked potential (ssVEP, Müller et al., 1998). The ssVEP is an oscillatory neuronal response to stimuli that are periodically modulated in luminance. Heightened ssVEP amplitudes mark increased visuocortical activation and can be used to index attentional processes. For example, attended features (Müller et al., 2006) and selective spatial attention (Müller et al., 1998) facilitate visuocortical activation compared to unattended features and locations. SsVEPs are also sensitive to emotional

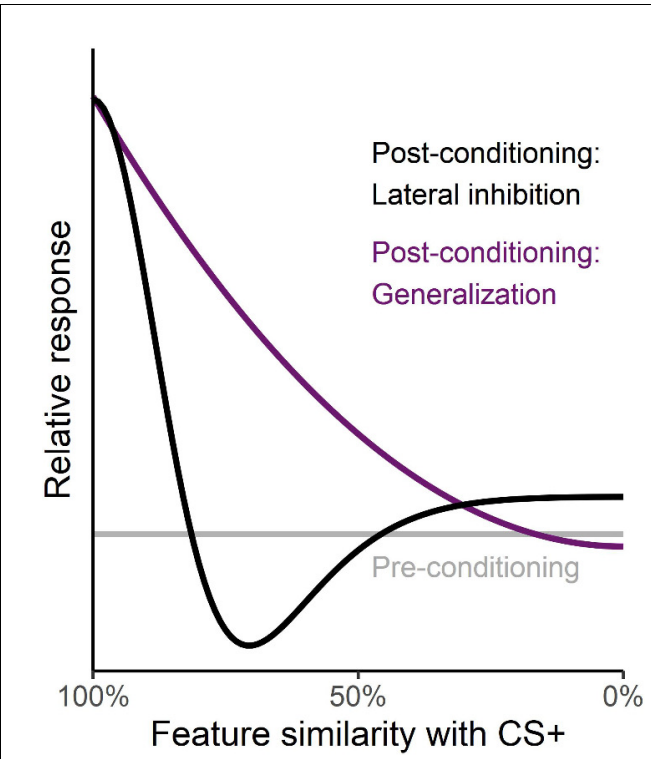

Figure 1. Different tuning functions during aversive learning. The flat grey line depicts relative (behavioral or neural) responses along a physical feature gradient during pre-conditioning. The black curve shows a possible tuning function for lateral inhibition after aversive conditioning as seen in orientation-selective neuronal populations in the visual cortex (McTeague et al., 2015). Relative responses are enhanced for the specific feature associated with the aversive event and supressed for the most similar features. In contrast, the purple curve depicts a gradually decreasing generalization gradient observed in self-report and somato-visceral indices of generalization learning. processes and show increased amplitudes for emotional compared to neutral stimuli (Keil et al., 2003; Kemp et al., 2002; McTeague et al., 2011). Therefore, they provide a promising method for testing hypotheses regarding changing perception and attention as participants undergo fear generalization learning (Wieser et al., 2016). The amplitude of the ssVEP differentiates threat from safety signals, being selectively heightened for conditioned threat cues (reviewed in Miskovic and Keil, 2012). Building on these findings, McTeague et al., 2015 utilized ssVEPs to study population-level tuning of orientation-selective neurons in the primary visual cortex during fear generalization. In the neuroscience literature, tuning functions are often used to to describe differences in sensitivity of a response (neural or behavioral) along a physical feature gradient. For example, orientation tuning functions denote how neurons in the retinotopic visual cortex selectively respond to specific orientations (see Figure 1). At the population level, especially with scalp record fields, information regarding the tuning of individual neurons is obscure. Changes in the preferential tuning of population-level responses along a feature gradient can however be assessed with suitable research designs: To examine the extent to which aversive learning affects the population-level orientation tuning reflected in ssVEPs, McTeague et al., 2015 used high-contrast grating stimuli differing in orientation as conditioned stimuli. During pre- 
conditioning, the sSVEP amplitude evoked by each orientation was the same, resulting in a flat tuning curve. During acquisition, only the grating stimulus in the middle of this stimulus continuum was paired with an aversive noise (i.e. the $\mathrm{CS}+$ ). After several conditioning trials, the CS+ prompted enhanced visuocortical engagement, accompanied by a suppression of the grating orientations with highest similarity to the CS+. This tuning pattern, which contrasts with the gradually decreasing generalization gradient observed in self-report and somato-visceral indices of learning, suggests lateral inhibitory interactions among orientation-selective neuronal populations in the visual cortex.

In the present study, we tested the hypothesis that sensory systems, when presented with a similarity gradient around a social threat stimulus, undergo changes to sharpen their tuning properties toward the relevant feature. Paralleling work on orientation-selective neurons discussed above, we expected amplification of visuocortical responses to the threat-associated face and a selective suppression of responses to the face most similar to the threat-associated face, reflecting inhibitory interactions between neuronal populations that represent facial features. This hypothesis is grounded in work showing substantial evidence for single-unit and population level (LFPs, fMRI) tuning in face-specific areas in the human and primate brain (Freiwald and Tsao, 2010; Freiwald et al., 2009; Gilaie-Dotan and Malach, 2007; Leopold et al., 2006; Loffler et al., 2005). These studies have demonstrated that there are neurons and neuronal populations in face-sensitive cortical areas, like the occipital face area (OFA) and the fusiform face area (FFA), which show gradual responses to varying facial identify, often referred to as 'tuning' to facial identities (Chang and Tsao, 2017). Here, we examine the malleability of population-level tuning as observers learn to associate one identity along a gradient of morphs with an aversive outcome. To further establish the behavioral relevance of visuocortical tuning, we also examine the extent to which such sharpened visuocortical tuning is associated with interindividual differences in social anxiety.

\section{Results}

\section{Habituation}

Steady-state visually evoked potentials (ssVEPs) To induce ssVEPs, two different facial stimuli (CS+ and CS-) were presented with a flickering frequency of $12 \mathrm{~Hz}$ (see Figure 2). After converting the electrocortical signal to current source density (CSD) estimates and transforming it into the frequency-domain, the signal-to-noise ratio (SNR) was calculated by dividing the power of the driving frequency by the mean of the spectral power at six adjacent frequency bins, leaving out the two immediate neighbors. The resulting SNRs were pooled across eight sensor locations over the occipital pole for statistical analyses. During habituation, the linear mixed model analysis including the within-factor CS-type (two levels: CS+ vs CS-) and social anxiety as a continuous between-variable revealed a significant main effect for social anxiety, indicating that higher social anxiety was associated with higher ssVEP-SNRs in general and a non-significant trend for CS-type (CS+: $M=3.61, S D=1.91$; $\left.C S-: M=3.39 S D_{=1} 1.77\right)$. There was no CS-type $x$ social anxiety interaction (see Table 1).

\section{Valence and arousal ratings}

The linear mixed model analysis of valence ratings revealed neither a significant main effect of social anxiety nor a CS-type $x$ social anxiety interaction (see Table 1). However, subjects rated the CS- as slightly more unpleasant than the $C S+,\left(C S+: M=4.60, S D=1.22 ; C S-: M=4.99 S D_{=} 1.25\right)$. With regard to arousal ratings, there was no significant effect for $C S$-type $(C S+: M=3.69, S D=1.61$; $C S$-: $\left.M=3.58 S D_{=} 1.58\right)$, social anxiety, or CS-type $x$ social anxiety interaction. This result points out that none of the two faces elicited more arousal at the beginning of the experiment.

\section{Acquisition}

\section{SsVEPs}

After the CS+ had been paired with the US (see Figure 2), linear mixed model analyses for ssVEPs yielded a main effect of CS-type and a small effect of social anxiety, demonstrating that subjects reacted with higher amplitudes to the $C S+(M=3.69, S D=1.80)$ compared to the $C S-(M=3.33$, $S D=1.56$, see Figure $3 a$ ) and that higher social anxiety was associated with higher amplitudes in general (see Table 2). There was no significant CS-type $x$ social anxiety interaction. 


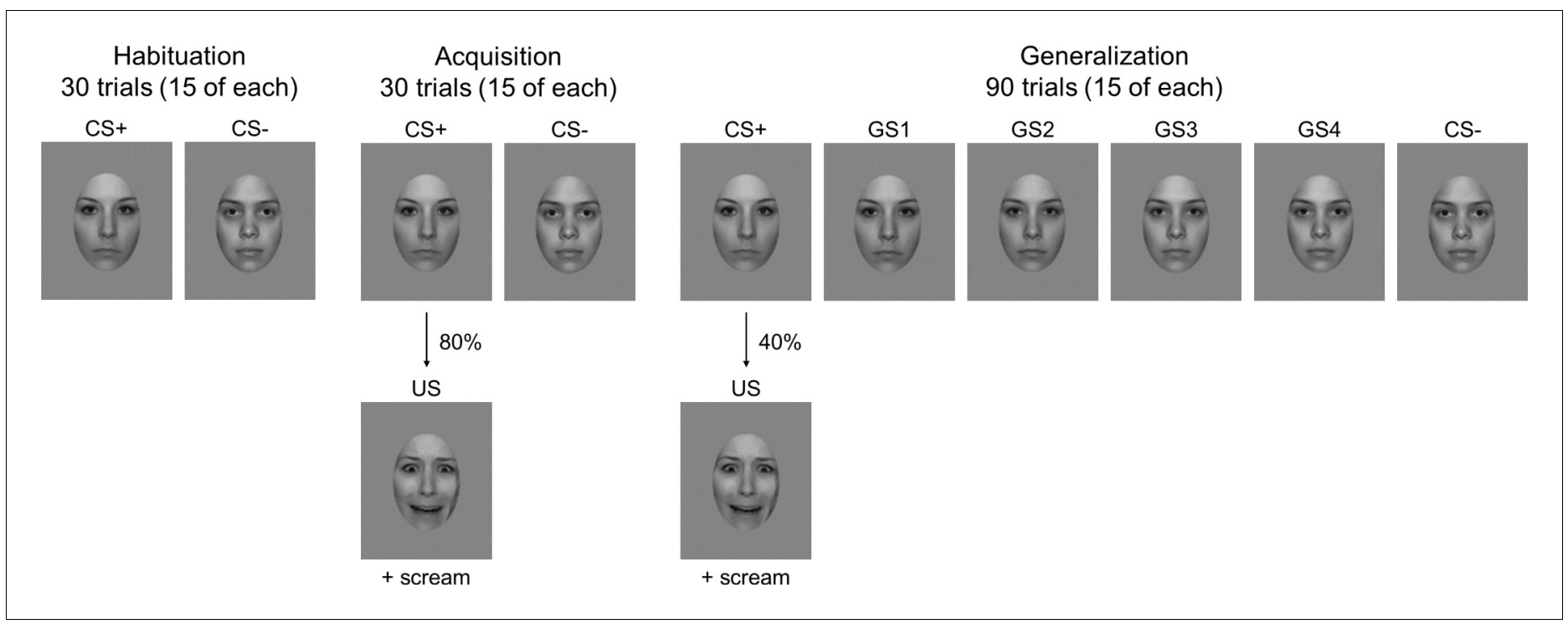

Figure 2. Experimental design. Habituation, acquisition and generalization phase are illustrated. Stimuli were randomly presented for $3 \mathrm{~s}$ during each of the three parts of the experiment. The US consisted of a $1500 \mathrm{~ms}$ presentation of the respective CS+ face displaying a fearful expression, which was accompanied by a $95 \mathrm{~dB}$ shrill female scream. The assignment of face to CS+/CS- was counterbalanced between participants.

Valence and arousal ratings

After acquisition, there was a significant main effect of CS-type for both valence and arousal ratings (see Table 2), demonstrating that subjects rated the $C S+$ as more unpleasant $(M=6.42, S D=1.41)$ and more arousing $(M=6.51, S D=1.63)$ compared to the $C S$ - (unpleasantness: $M=4.73, S D=1.34$; arousal: $M=3.69, S D=1.94$, see Figure $3 c$ and $d$ ). Main effects of social anxiety and CS-type $x$ social anxiety interactions were not significant.

\section{US expectancy rating}

The analysis detected a main effect of CS-type (see Table 2), with higher US expectancy ratings for the $C S+(M=72.09, S D=21.71)$ compared to the $C S-(M=8.66, S D=18.74)$, underlining that the experimental manipulation was effective (see Figure $3 \mathrm{~b}$ ). There was no effect of social anxiety or CS-type $x$ social anxiety interaction.

Table 1. Results of the linear mixed model analyses during habituation. sSVEP-SNRs:

\begin{tabular}{lllll}
\hline CS-Type & $F(1,65)=3.18$ & $p=0.079$ & $R^{2}=0.047$ & $\mathrm{Cl}=[.000, .187]$ \\
\hline Social anxiety & $t(65)=2.18$ & $p=0.033$ & $\beta=0.48$ & $\mathrm{SE}=0.21$ \\
\hline CS-Type x Social anxiety & $F(1,65)=0.69$ & $p=0.408$ & $R^{2}=0.011$ & $\mathrm{Cl}=[.000, .112]$ \\
\hline Valence: & & & \\
\hline CS-Type & $F(1,65)=4.07$ & $p=0.048$ & $R^{2}=0.059$ & $\mathrm{Cl}=[.001, .207]$ \\
\hline Social anxiety & $t(65)=1.34$ & $\mathrm{p}=0.186$ & $\beta=-0.15$ & $\mathrm{SE}=0.12$ \\
\hline CS-Type x Social anxiety & $F(1,65)=1.16$ & $\mathrm{p}=0.285$ & $R^{2}=0.018$ & $\mathrm{Cl}=[.000, .130]$ \\
\hline Arousal: & & & \\
\hline CS-Type & $F(1,65)=0.34$ & $p=0.562$ & $R^{2}=0.005$ & $\mathrm{Cl}=[.000, .096]$ \\
\hline Social anxiety & $t(65)=1.24$ & $p=0.221$ & $\beta=0.21$ & $\mathrm{SE}=0.17$ \\
\hline CS-Type x Social anxiety & $F(1,65)=2.18$ & $\mathrm{p}=0.145$ & $R^{2}=0.032$ & $\mathrm{Cl}=[.000, .162]$ \\
\hline
\end{tabular}




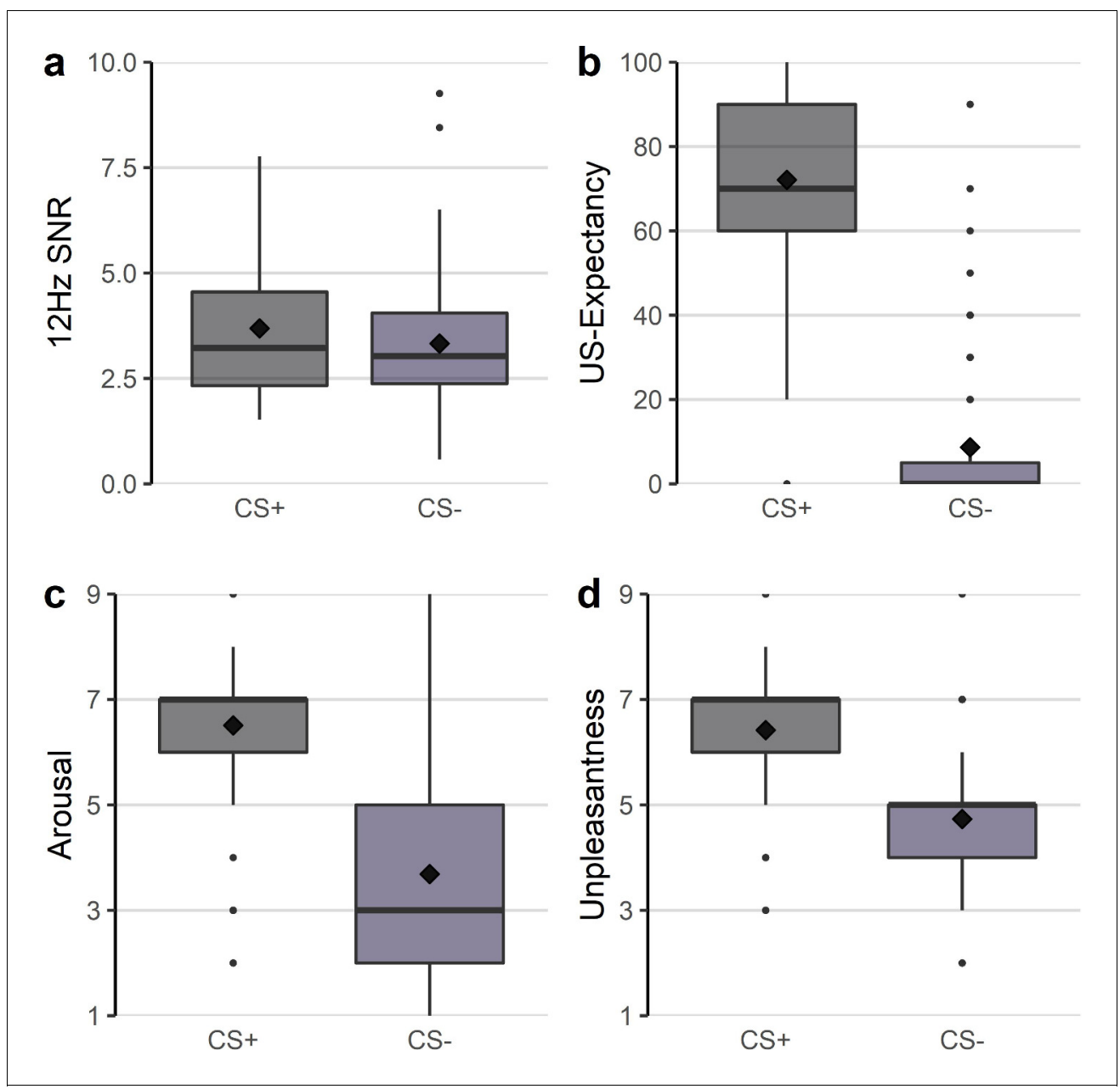

Figure 3. Boxplots and means (diamonds) of the (a) $12 \mathrm{~Hz}$ ssVEP signal-to-noise ratios (SNR) during the acquisition phase and mean US expectancy (b), arousal (c), and unpleasantness ratings (d) after acquisition.

\section{Generalization}

Steady-state visually evoked potentials

During the generalization test, four additional faces (GS1 - 4) were presented by morphing the two faces of the previous phases together in $20 \%$ steps, with the GS1 being the most similar to the CS+ and the GS4 being the most similar to the CS- (see Figure 2). The CS-type (six levels: CS+ vs GS1 vs GS2 vs GS3 vs GS4 vs CS-) x social anxiety linear mixed model provided a main effect of CS-type and a marginal effect of social anxiety (see Figures 4 and $5 \mathrm{a}$ and Table 3). Post-hoc contrasts indicated significant differences between GS4 vs CS-, $t(325)=2.94, p=0.003$, GS3 vs CS-, $t(325)=3.24$, $p=0.001$, and CS + vs CS-, $t(325)=2.17, p=0.031$, but not between GS2 vs CS-, $t(325)=1.75$, $p=0.081$, and GS1 vs CS-, $t(325)=0.82, p=0.411$. However, the omnibus-test revealed no stimulus type $x$ social anxiety interaction.

To follow up on the frequentist statistics and to seek converging evidence from a Bayesian perspective, we compared the fit of a lateral inhibition pattern to a quadratic and linear trend, using weighted predictors in Bayesian linear models (see Figure 6). To this end, we constructed weight vectors that reflected the predictions of the alternative hypotheses for the experimental conditions. This approach allowed us to leverage the specific model predictions under the lateral inhibition, generalization, and linear hypotheses, and to quantify the fit between the empirical data and model predictions in one test across all conditions. The lateral inhibition pattern was expressed as the difference of two Gaussians (weights: $+2,-2,+0.5,+1,+0.5,-2$ for CS+, GS1, GS2, GS3, GS4, CS-), paralleling previous studies on visuocortical tuning (Antov et al., 2020; McTeague et alo, 2015). 
Table 2. Results of the linear mixed model analyses during acquisition learning. SSVEP-SNRs:

\begin{tabular}{|c|c|c|c|c|}
\hline CS-Type & $F(1,65)=5.50$ & $p=0.022$ & $R^{2}=0.078$ & $C l=[.003, .235]$ \\
\hline Social anxiety & $t(65)=2.00$ & $p=0.050$ & $\beta=0.37$ & $S E=0.19$ \\
\hline CS-Type $x$ Social anxiety & $F(1,65)=1.57$ & $p=0.168$ & $R^{2}=0.013$ & $\mathrm{Cl}=[.000, .118]$ \\
\hline \multicolumn{5}{|l|}{ Valence: } \\
\hline CS-Type & $F(1,65)=49.51$ & $p<0.001$ & $R^{2}=0.432$ & $\mathrm{Cl}=[.271, .587]$ \\
\hline Social anxiety & $t(65)=0.80$ & $p=0.424$ & $\beta=0.09$ & $S E=0.12$ \\
\hline CS-Type $\times$ Social anxiety & $F(1,65)=0.00$ & $p=0.982$ & $R^{2}=0.000$ & $\mathrm{Cl}=[.000, .075]$ \\
\hline \multicolumn{5}{|l|}{ Arousal: } \\
\hline CS-Type & $F(1,65)=91.13$ & $p<0.001$ & $R^{2}=0.584$ & $\mathrm{Cl}=[.447, .705]$ \\
\hline Social anxiety & $t(65)=0.05$ & $p=0.959$ & $\beta=0.01$ & $S E=0.16$ \\
\hline CS-Type x Social anxiety & $F(1,65)=1.29$ & $p=0.259$ & $R^{2}=0.020$ & $\mathrm{Cl}=[.000, .135]$ \\
\hline \multicolumn{5}{|l|}{ US expectancy: } \\
\hline CS-Type & $F(1,65)=323.15$ & $p<0.001$ & $R^{2}=0.833$ & $\mathrm{Cl}=[.771, .884]$ \\
\hline Social anxiety & $t(65)=0.17$ & $p<0.867$ & $\beta=-0.30$ & $S E=1.77$ \\
\hline CS-Type $\times$ Social anxiety & $F(1,65)=0.15$ & $p=0.736$ & $R^{2}=0.002$ & $\mathrm{Cl}=[.000, .083]$ \\
\hline
\end{tabular}

The quadratic (weights: $+2.5334,+1.0934,-0.0267,-0.8267,-1.3067,-1.4667$ ) and linear (weights: $+2.5,+1.5,+0.5,-0.5,-1.5,-2.5)$ trend were modeled after the analyses of the linear and quadratic component of the generalization gradient, which are commonly employed in the fear generalization literature (Ahrens et al., 2016; Lissek et al., 2014a; Lissek et al., 2014b). In a first step, each Bayesian model was compared to the 'random intercept only' model (null model, 0), before transitive Bayes factors were calculated to obtain the relative evidence of one model over another. These transitive Bayes factors allow a direct comparison between the competing models. For a summary of resulting Bayes factors for each candidate main effect, interaction and predictor weight model see Table 4. The main effect of weighted CS-type received support for the lateral inhibition pattern only, $B F_{\mathrm{La} 1 / 0}=13.26$, but not for the quadratic, $B F_{\mathrm{Q} 1 / 0}=0.14$, or linear trend, $B F_{\mathrm{Li} 1 / 0}=0.13$. Further including a main effect of social anxiety did not lead to substantially increased support for the lateral inhibition pattern, $B F_{\mathrm{La} 2 / \mathrm{La} 1}=1.38$, the quadratic, $B F_{\mathrm{O} 2 / \mathrm{O} 1}=1.08$, or linear trend, $B F_{\mathrm{Li} 2 / \mathrm{Li} 1=1.36}$. The strongest evidence could be found for the full interaction model (SNR CS type + social anxiety + CS-type $x$ social anxiety) and the lateral inhibition pattern, $B F_{\text {La3 } / 0}=57.32$, which substantially extends the support of the main effects model, $B F_{\mathrm{La} 3 / \mathrm{La} 2}=3.13$, suggesting that the accentuation of the lateral inhibition pattern increased with higher social anxiety. By contrast, the full interaction models for the Quadratic, $B F_{\mathrm{Q} 3 / 0}=0.11$, and Linear trend, $B F_{\mathrm{Li} / 0}=0.38$, still yielded less evidence

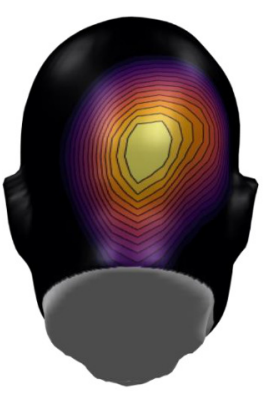

$\mathrm{CS}+$

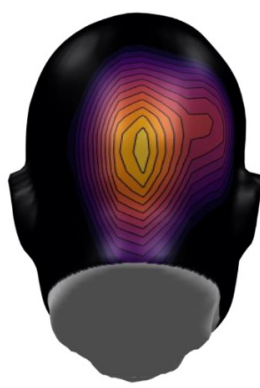

GS1

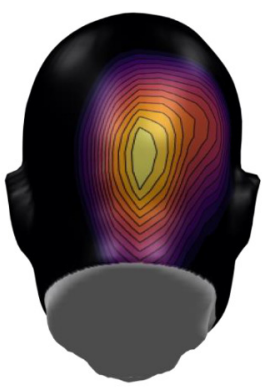

GS2

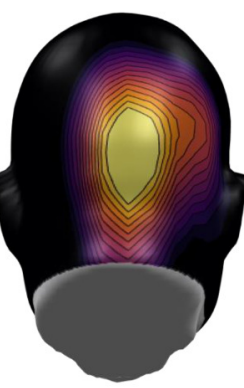

GS3

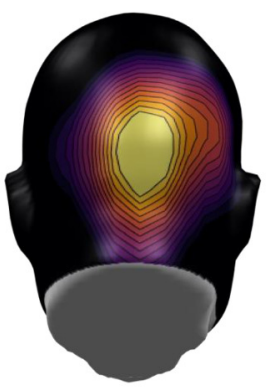

GS4

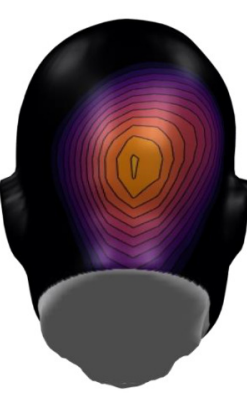

CS-

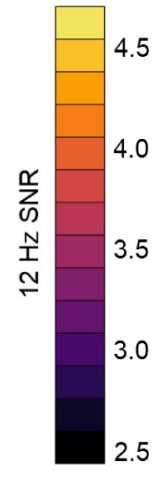

Figure 4. Mean scalp topographies of the $12 \mathrm{~Hz}$ ssVEP signal-to-noise ratios to the conditions during the generalization test. 


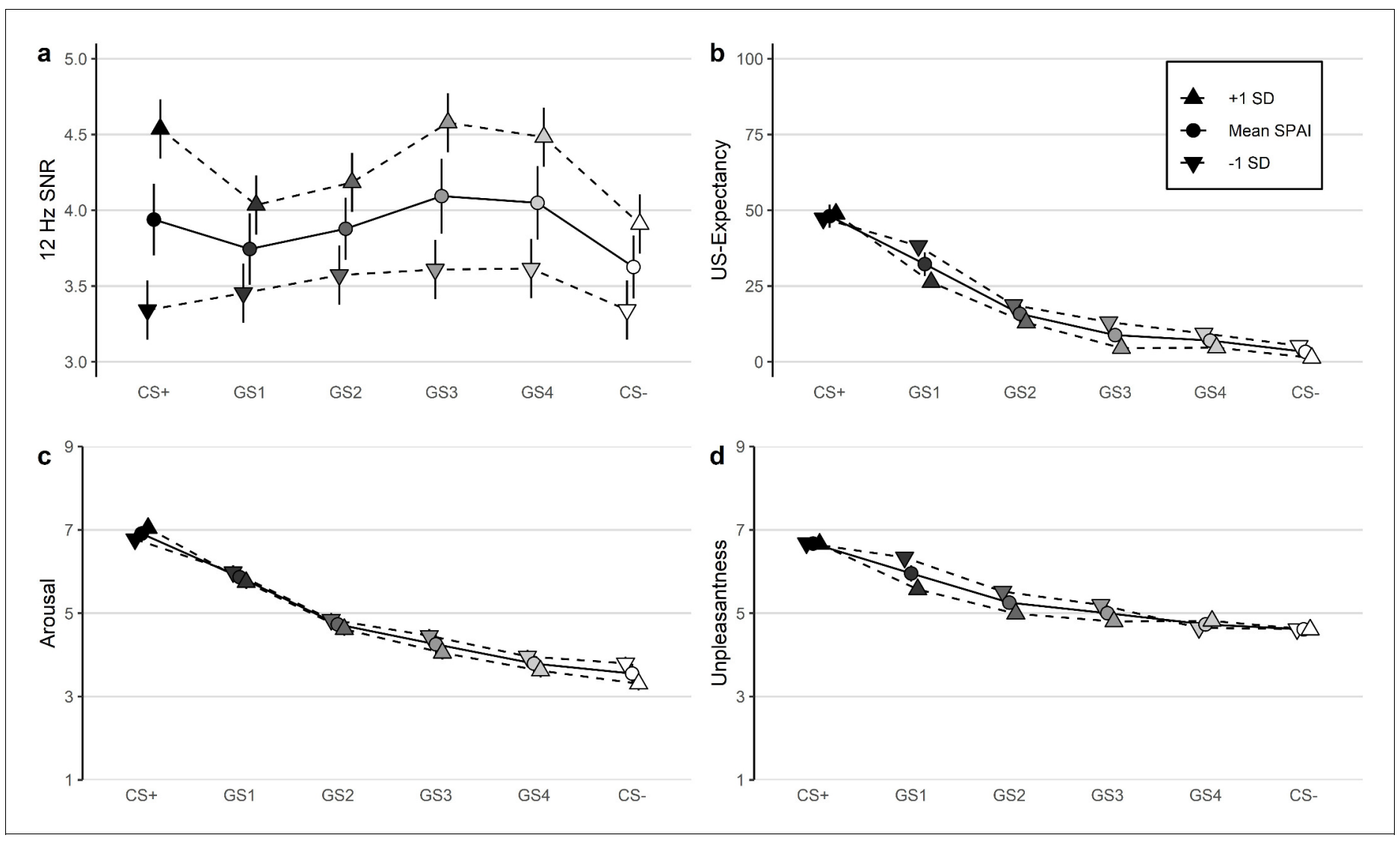

Figure 5. Generalization test: (a) Mean $12 \mathrm{~Hz}$ signal-to-noise ratios (SNR) $\pm S E M$ of the ssVEP during the generalization test. (b) Mean US-expectancy, (c) arousal and, (d) unpleasantness ratings \pm SEM after generalization. Prediction intervals are shown for the mean \pm 1 SD of the SPAI covariate to illustrate the influence of social anxiety. 
Table 3. Results of the linear mixed model analyses during generalization learning. SSVEP-SNRs:

\begin{tabular}{|c|c|c|c|c|}
\hline CS-Type & $F(5,325)=3.39$ & $p=0.009$ & $R^{2}=0.045$ & $\mathrm{Cl}=[.020, .111]$ \\
\hline Social anxiety & $t(65)=1.94$ & $p=0.056$ & $\beta=0.40$ & $S E=0.21$ \\
\hline CS-Type x Social anxiety & $F(5,325)=1.57$ & $p=0.167$ & $R^{2}=0.024$ & $\mathrm{Cl}=[.009, .080$ \\
\hline \multicolumn{5}{|l|}{ Valence: } \\
\hline CS-Type & $\begin{array}{l}F(5,325) \\
=35.83\end{array}$ & $p<0.001$ & $R^{2}=0.355$ & $\mathrm{Cl}=[.286, .436]$ \\
\hline Social anxiety & $t(65)=0.17$ & $p<0.867$ & $\beta=0.13$ & $S E=0.12$ \\
\hline CS-Type $x$ Social anxiety & $F(5,325)=1.83$ & $p=0.107$ & $R^{2}=0.027$ & $\mathrm{Cl}=[.011, .086]$ \\
\hline \multicolumn{5}{|l|}{ Arousal: } \\
\hline CS-Type & $\begin{array}{l}F(5,325) \\
=66.80\end{array}$ & $p<0.001$ & $R^{2}=0.507$ & $\mathrm{Cl}=[.443, .574]$ \\
\hline Social anxiety & $t(65)=1.12$ & $p=0.267$ & $\beta=-0.12$ & $S E=0.18$ \\
\hline CS-Type $\times$ Social anxiety & $F(5,325)=0.71$ & $p=0.618$ & $R^{2}=0.011$ & $C l=[.005, .060]$ \\
\hline \multicolumn{5}{|l|}{ US expectancy: } \\
\hline CS-Type & $\begin{array}{l}F(5,325) \\
=57.30\end{array}$ & $p<0.001$ & $R^{2}=0.469$ & $C l=[.402, .540]$ \\
\hline Social anxiety & $t(65)=1.37$ & $p=0.174$ & $\beta=-2.79$ & $S E=2.03$ \\
\hline CS-Type $\times$ Social anxiety & $F(5,325)=0.97$ & $p=0.435$ & $R^{2}=0.015$ & $\mathrm{Cl}=[.006, .067]$ \\
\hline
\end{tabular}

Valence and arousal ratings

The linear mixed models of the ratings yielded a significant main effect of CS-type for both valence and arousal ratings (see Table 3). Following the procedure of the ssVEP analysis, follow-up contrasts were calculated. With regard to arousal (see Figure $5 \mathrm{c}$ ), subjects differentiated among the CS- and the CS+ plus three GS: CS- versus CS+, $t(325)=14.93, p<0.001, C S$ - versus $G S 1, t(325)=10.28$, $p<0.001, C S$ - versus GS2, $t(325)=5.24, p<0.001$, and CS- versus $G S 3, t(325)=3.12, p=0.002$, but not CS- versus $G S 4, t(325)=1.06, p=0.290$. For valence ratings (see Figure $4 d$ ), results showed differences between CS- and CS $+, t(325)=10.95, p<0.001, C S-$ and $G S 1, t(325)=7.14, p<0.001, C S-$ and GS2, $t(325)=3.41, p<0.001$, and CS- and GS3, $t(325)=2.06, p=0.040$, but not between CSand GS4, $t(325)=.64, p=0.526$. These results suggest that all subjects transferred their fear response reflected in both valence and arousal ratings from the CS+ to three GSs. In addition, there were no main effects of social anxiety nor CS-type $x$ social anxiety interactions.

\section{US expectancy ratings}

US expectancy analysis revealed a main effect of CS-type (see Figure 5b and Table 3). Post-hoc contrasts yielded significant effects for the comparison of CS- versus CS $t(325)=13.73, p<0.001 ; G S 1$ : $t(325)=8.88, p<0.001$; and GS2: $t(325)=3.84, p<0.001$. The differences between CS- and GS3: $t$ $(325)=1.69, p=0.091$, and CS- and GS4, $t(325)=1.14, p=0.254$, were not significant. As found in valence and arousal ratings, subjects showed an enhanced tendency to generalize their conditioned fear reaction, indicated by the fact that they expected the GS1 and GS2 to be followed by the US, although they had never been paired with the US. The main effect of social anxiety and the CS-type $x$ social anxiety interaction were not significant.

\section{Discussion}

The goal of the present study was to test the hypothesis that aversive generalization learning prompts sharpened representations of facial identity, reflecting inhibitory interactions between neuronal populations that represent facial features associated with threat versus safety. Second, we leveraged interindividual differences in social anxiety to examine whether the sharpened visuocortical tuning to facial identity is heightened in those characterized by higher social anxiety. For this 

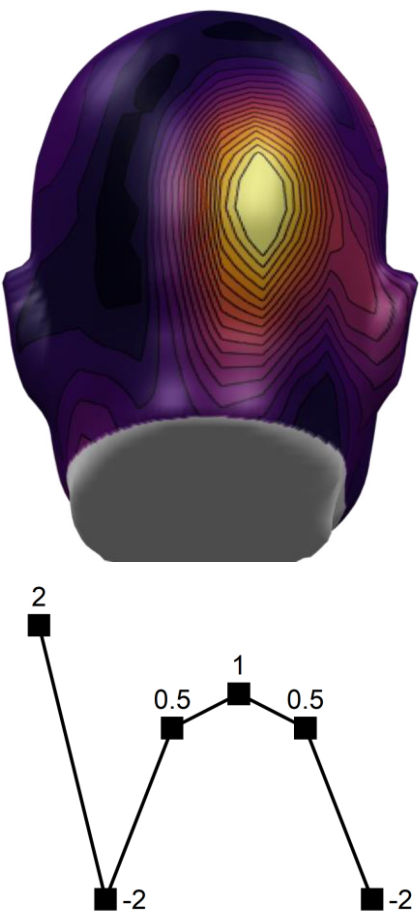

CS+ GS1 GS2 GS3 GS4 CSModel: Lateral inhibition
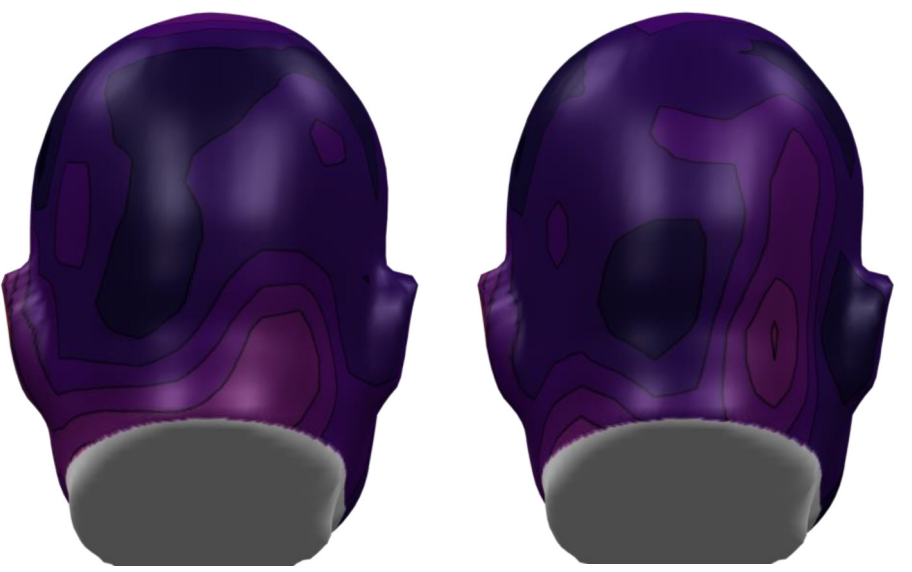

$\operatorname{Ln}\left(\mathrm{BF}_{1 / 0}\right)$

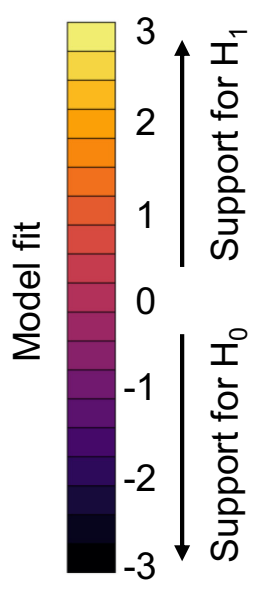

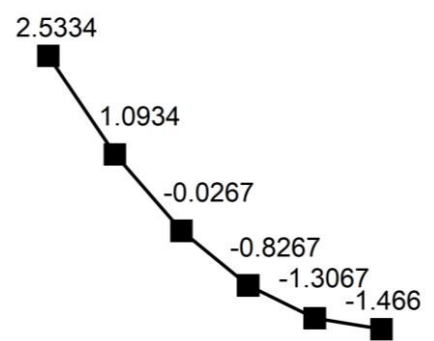

CS+ GS1 GS2 GS3 GS4 CSModel: Quadratic trend

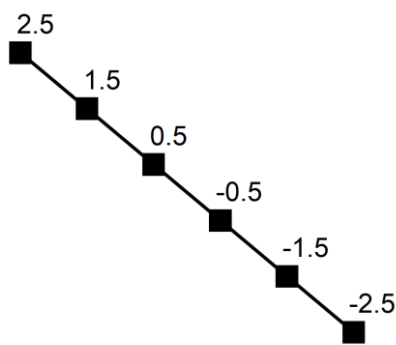

CS+ GS1 GS2 GS3 GS4 CSModel: Linear trend

Figure 6. Bayesian model fit: Topographical distributions of the Bayes Factor for comparing the main effect model 'SNR CS-type' to the null model for each contrast. Weights used for the contrasts are displayed at the bottom row. Natural log-transformed BFs are illustrated, so that positive values display support for the full effect model while negative values display support for the null model.

purpose, steady-state visually evoked potentials (ssVEPs) as well as valence, arousal and US expectancy ratings were recorded in a fear conditioning and generalization paradigm with social cues.

Crucial for the later generalization test, successful fear conditioning was indexed in both ssVEP amplitude and arousal, valence and US expectancy ratings during acquisition, and social anxiety was not associated with stronger discrimination between conditioned stimuli or generally increased ratings. Thus, the CS+ during acquisition elicited increased visuocortical responses reflecting enhanced sensory engagement (Stegmann et al., 2019a; Wieser et al., 2014c). However, during habituation and acquisition, subjects with higher social anxiety showed overall amplified visuocortical responses to the face stimuli, which is in line with the notion of generally heightened sensitivity to facial expressions in social anxiety disorder (McTeague et alo, 2018) and has been observed previously in

Table 4. Summary of the Bayesian linear model analysis.

\begin{tabular}{lllllll} 
BF $_{\mathbf{M} / 0}$ & Model & Lateral inhibition & Quadratic trend & Linear trend & Inhibition vs quadratic & $\begin{array}{l}\text { Inhibition vs } \\
\text { Linear }\end{array}$ \\
\hline$M_{1}:$ & SNR $\sim$ CS-type & 13.26 & 0.14 & 0.13 & 104.17 & 97.62 \\
\hline$M_{2}:$ & SNR $\sim$ CStype + SA & 18.33 & 0.15 & 0.17 & 105.49 & 125.16 \\
\hline$M_{3}:$ & SNR $\sim$ CS type + SA + CS-type x SA & 57.32 & 0.11 & 0.38 & 858.52 & 502.184 \\
\hline
\end{tabular}

Bayes factors of main and interaction effect models $\left(M_{1}-M_{3}\right)$ compared to the 'random intercept only' model (Null model) for the lateral inhibition pattern (weights: $+2,-2,+0.5,+1,+0.5,-2$ for CS+, GS1, GS2, GS3, GS4, CS-), quadratic trend (weights: $+2.5334,+1.0934,-0.0267,-0.8267,-1.3067,-1.4667$ ) and linear trend (weights: $+2.5,+1.5,+0.5,-0.5,-1.5,-2.5)$. The last two columns display direct model comparisons between the lateral inhibition pattern to the quadratic or linear trend by dividing respective BFs for each main and interaction effect model. SA, social anxiety. 

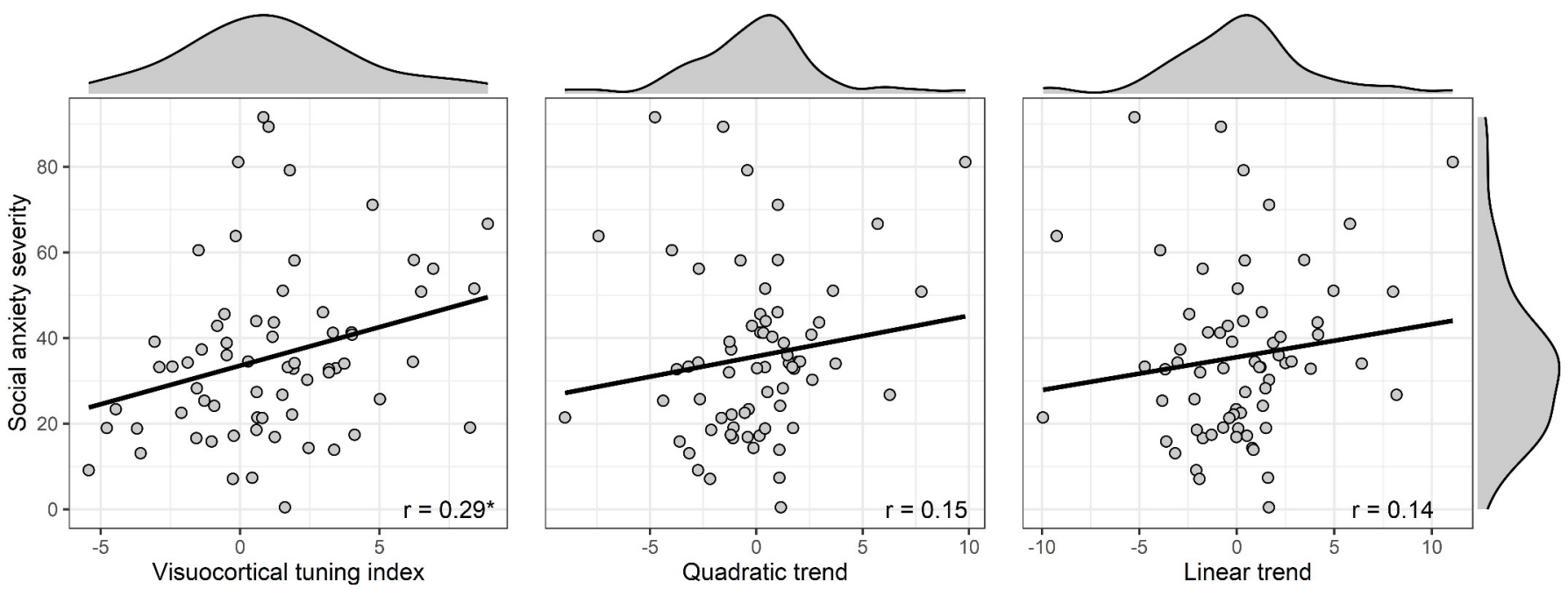

Figure 7. Comparison of the correlations between social anxiety and the different contrast models. Only the visuocortical tuning index as a parameter for the accentuation of the lateral inhibition pattern significantly increased with social anxiety. Marginal density plots display the distributions of the social anxiety scores and the ssVEP indices.

patients with SAD using ssVEPs (McTeague et al., 2011) and in high socially anxious individuals using ERP components (Mühlberger et alo, 2009) as markers of early perceptual processing.

During fear generalization, ratings of arousal, valence and US expectancy monotonically diminished with decreasing similarity to the CS+, indicating that subjects transferred their fear response from the threat signaling face to similar faces, although those had not been associated with the aversive outcome. These results corroborate previous studies on conditioned generalization, which demonstrated gradual, monotonic, generalization effects for subjective ratings (Lissek et al., 2008; McTeague et al., 2015; Stegmann et al., 2019b), somato-visceral measures, such as fear-potentiated startle (Lissek et alo, 2008), skin conductance responses (Stegmann et alo, 2019b; TorrentsRodas et al., 2013) and heart rate (Ahrens et al., 2016) and electrocortical responses, that is, late positive potentials (Nelson et al., 2015).

Importantly, the visuocortical responses did not show a monotonic generalization gradient, but instead displayed a response pattern consistent with sharpening of the threat face representation. The amplitude of the ssVEPs - in contrast to the ratings - did not gradually diminish with increasing distance from the CS+. Instead, ssVEP amplitude for the CS+ was increased, but followed by an immediate reduction for the closest GS (GS1), and a slow increase in response to the remaining GSs, before it was again reduced for the learned safety cue (CS-). This observation is consistent with the response pattern of orientation sensitive neurons in the visual cortex during a fear generalization paradigm with enhanced visuocortical engagement to the CS+ and a suppression of the grating orientations with the highest similarity to the CS+ (McTeague et alo, 2015). In line, the Bayesian analyses demonstrated that the observed ssVEP generalization data more likely corresponds to the lateral inhibition model compared to the null model, which received more support from the Bayesian analyses than the quadratic or linear trend model.

Together, these findings suggest that there is a dissociation in the aversive generalization patterns of sensory compared to efferent and autonomic systems, which is consistent with the idea that fear generalization is an active and multifaceted process that integrates a wide variety of signals to organize adaptive fear responses (Onat and Büchel, 2015). A large body of work has shown that visuocortical engagement with specific stimulus features varies with the motivational relevance of these features (Bradley et al., 2012). The present results are in line with this notion, but also suggest that these adaptive sensory changes observed during learning differ from the efferent system's responses. To date, there are not many studies that are directly targeting the functional differences between sensory and efferent systems. Using steady-state visual evoked fields (the 
magnetoencephalographic counterpart of ssVEPs), two studies could demonstrate that threatinduced sensory changes in low-level visual areas occur independently of the conscious awareness of the CS-US contingencies (Moratti and Keil, 2009; Yuan et al., 2018), suggesting that visuocortical responses are to some extent independent of higher level cognitive processes. In contrast, it has been suggested, that threat-induced changes in the sensory system represent short-term plasticity in the early visual cortex, which might be induced by projections from subcortical structures like the amygdala or thalamus (Miskovic and Keil, 2012). This could be one reason for the divergence between visuocortical and subjective responses, as verbal reports reflect a more cognitive component of the defensive response (LeDoux and Pine, 2016). On the other hand, McTeague et al., 2015 also found a dissociation between the visuocortical and the fear-potentiated startle responses. The startle reflex, as an index of the autonomic component of the efferent system, is also assumed to be directly modulated by amygdala projections (Davis, 2006). In this case, the discrepancies between sensory and efferent response patterns might be mediated by different subregions of the amygdala, which modulate the sensory and efferent system according to their supposed functions in threat detection and defensive responding. Taking an evolutionary perspective, it is most adaptive for the organism to enhance sensory specificity in the visual cortex to distinguish the motivational information-providing stimulus from others sensory signals as reflected in the lateral inhibition model. On the other hand, the 'efferent' readiness to respond to a potential threat is generalized, as reflected in a monotonically decreasing generalization gradient, because a false alarm is less costly than a - potentially fatal - miss. This evolutionary interpretation assumes that the constantly changing and diverse environment in which humans find themselves demands and thus favors plastic physiological mechanisms, which may differ between systems in order to optimize functioning (Miskovic and Keil, 2012).

The present study also has implications regarding the neural mechanisms mediating aversive generalization learning, and regarding the neocortical changes underlying learning and memory more broadly: McTeague et al., 2015, using a generalization gradient of oriented gratings, explained their finding of visuocortical sharpening as a consequence of lateral inhibitory interactions among orientation-selective neuronal populations in the primary visual cortex. In this case, signals from frontoparietal attention networks may selectively facilitate CS+ representations in visual cortex, prompting local inhibitory interactions between adjacent cortical units. This process is thought to prompt suppression of the features represented by the most spatially proximal populations. In fact, ongoing computational modeling efforts in our laboratories explain these and other generalization data best by assuming that top-down signals take the shape of a generalization gradient (paralleling behavioral and autonomic data), and it is the organization of visual cortex that turns this gradient into a sharpening pattern through lateral inhibition. As such, sharpening would not be inherited by anterior areas but would result from the organization and geometry of visual cortical areas. This interpretation is further supported by research demonstrating that the orientation-tuning functions of visual neurons may be shaped by short-term plastic processes (Dragoi et al., 2000).

We hypothesized that in a situation in which facial identity predicts threat versus safety, a similar mechanism may operate in the cortical tissue that is specific not to orientation but to the features encoding facial identity. There are two loci where such tuning may occur: First, as aversive learning proceeds, projections from anterior structures may increasingly target lower-level representations of individual facial features in retinotopic areas, thus prompting inhibitory interactions between individual physical facial features such as orientation or spatial frequency, which differ across the morphing gradient. This is consistent with findings and models in perceptual learning (e.g. reverse hierarchy theory; Ahissar and Hochstein, 2004) and would prompt a mid-occipital topography of sharpening effects in the present study.

Second, inhibitory interactions may occur between similar faces along a gradient of morphs, in face-sensitive cortical areas. This alternative hypothesis is in line with recent evidence suggesting that neuronal populations in face-sensitive cortical patches encode identity by combining their population tuning to sets of high-order shape and appearance dimensions (e.g. Chang and Tsao, 2017). Neural populations that are sensitive to such features may be located in the early visual cortex or at later stages of the face-processing pathway, for example the occipital face area (OFA) or the fusiform face area (FFA) (Duchaine and Yovel, 2015). The present study suggests that the amplification of these selective neuronal cell ensembles to a given threat face prompts lateral inhibitory interactions among neurons that are selective to slightly different facial features. Our results support the 
hypothesis that such interactions take place in extra-striate, higher order visuocortical areas, as we exploited the assets of high-density EEG recording and CSD-transformation leading to a precise topographical distribution of the ssVEP signal. These signal topographies reveal indeed right-lateralized activation peaks for the signal-to-noise ratios of the ssVEP signal (Figure 4) as well as for the model fit of the lateral inhibition pattern (Figure 6). Thus, our results are compatible with the idea of a right-hemispheric dominance of face perception (Rossion, 2014) and suggest an involvement of the right OFA or FFA in sharpening face discrimination on the basis of lateral inhibition.

Furthermore, Bayesian model and correlational analyses revealed that visuocortical system engagement is associated with self-reported symptoms of social anxiety. This result adds to a growing body of literature on attentional biases in social anxiety, including evidence from reaction-time tasks, (Bantin et alı, 2016), eye-tracking (Wieser et al., 2009), event-related potentials (ERPs, Mühlberger et al., 2009; Wieser et al., 2010) and ssVEPs (McTeague et al., 2018; McTeague et al., 2011). Here, we provide further evidence that cortical engagement in response to threat-associated phobic-relevant stimuli is dimensionally related to social anxiety. It is important to mention, however, that we - replicating Ahrens et al., 2016 - found no association between strength of social anxiety and (over)generalization of conditioned fear in terms of a higher fear responses (efferent system) to the generalization stimuli. Instead, we observed social anxiety to be associated with a more pronounced lateral inhibition pattern in visuocortical responses to generalization stimuli. Please note that the effect of social anxiety on visuocortical responses was only evident in the Bayesian-analysis but not in the corresponding ANOVA. This discrepancy results from the differences regarding statistical power between omnibus- and contrast-analysis (Furr and Rosenthal, 2003). The ANOVA tests for any differences, while the contrast-analysis only tests for deviations from the specified pattern. This notion is substantiated by the finding that social anxiety was not associated with visuocortical responses for the quadratic or linear trend model, but only for the lateral inhibition model. We conclude that this latter finding is indicative for the functional relevance of the lateral inhibition model in visuocortical tuning during generalization learning. Given the healthy subjects of this study, however, with those showing a psychiatric disorder being excluded, future studies should examine diagnosed and treatment-seeking SAD patients to substantiate initial findings and to draw conclusions on how sensory generalization versus sharpening contributes to the etiology or maintenance of social anxiety disorder or other psychopathologies.

One important limitation to our findings is that there was no substantial increase in differential ssVEP-SNRs (CS+ vs CS-) from habituation to acquisition, $\mathrm{t}(66)=0.83, \mathrm{p}=0.409, \mathrm{~d}=0.10, \mathrm{Cl}_{95}=$ $[-0.14,0.34]$. A reason for the lack of such a difference may be related to the trendwise differences between CS+ and CS- at baseline, which often indicate different responses to one of the two faces. However, this seems unlikely in our study, because the mapping of the two faces to the CS+ and CS- was fully counterbalanced between subjects. In addition, in the differential conditioning literature, cross-phase comparisons are typically avoided because they confound any conditioning effects with time-dependent effects such as adaptation, in which both the CS+ and CS- evoked responses decline over time, from habituation to acquisition and finally into the extinction phase. This has been well established in meta-analyses (e.g. Fullana et al., 2016), and is true for a wide range of dependent measures, including startle, skin conductance, fMRI, and ssVEPs where this pattern of findings has been noted and systematically addressed several times (e.g. Keil et al., 2013; Moratti and Keil, 2005). Thus, fear conditioning studies interested in temporal changes previously utilized analysis on a trial-by-trial level in order to avoid confoundation with these types of adaptation processes (e.g. Sjouwerman et al., 2016; Weike et al., 2007; Wieser et al., 2014c).

Post-hoc analyses comparing pre- and post-conditioning: although a lack of statistically robust changes between the experimental phases would not affect our interpretation of the main results regarding the generalization phase, we addressed this potential concern in a post-hoc analysis quantifying the amount of conditioning-related effects above and beyond the initial difference in habituation by means of parametric bootstrapping in combination with Bayesian analyses (Efron, 2012).

To this end, we computed a distribution for the CS+ versus CS- difference in ssVEP amplitude separately for habituation (to be used as prior distribution) and acqusition (posterior distribution) based on 100,000 bootstrapped group mean differences. Odds for conditioning effects to occur were estimated from these two distributions (the odds of the CS+-CS- difference to be positive), and the BF of interest was given as the ratio of posterior (acquisition) over prior (habituation) odds. The error of this process was estimated by repeating the above process 100 times and measuring 
the standard error of the resulting mean. This BF corresponds to the change in confidence that the ssVEP for the CS+ is greater than SsVEP for the CS- in acquisition (the posterior), relative to the prior distribution, which was estimated from the CS+ versus CS- difference during habituation. This analysis yielded a BF (posterior odds over prior odds) of 4.77 , error $=1.3 \%$, suggesting that the acquisition data provided substantial evidence for the notion that fear conditioning selectively amplifies the CS+ evoked ssVEP above and beyond the differences present during habituation.

In conclusion, our study extends current notions of generalization learning, by demonstrating the involvement of inhibitory interactions among feature-specific neurons in the visuocortical system during fear generalization to facial stimuli. We found that the accentuation of the lateral inhibition pattern increased with the severity of social anxiety. Future research may examine stability of the lateral inhibition response pattern during extinction as well as the role of perceptual sharpening in fear extinction learning.

\section{Materials and methods}

\section{Subjects}

Subjects were 67 undergraduate students (age: $M=24.10, S D=6.33$; 48 female) with normal or corrected vision and without past or present psychiatric diagnosis or family history of epilepsy (selfreport), who were paid or received course credit for participation. The sample size was based on the effect sizes of previous aversive conditioning studies using ssVEPs (McTeague et al., 2015; Miskovic and Keil, 2013) and adapted for covariate-analyses. Subjects completed the Social Phobia and Anxiety Inventory (SPAl, German version, Beidel et al., 1989) as a self-report measurement of social anxiety $(M=67.70, S D=19.74$, $M i n=32.48, M a x=126.60)$. Prior to participation, written informed consent was obtained from each participant. The study was approved by the ethics review board of the Medical Faculty of the University of Würzburg (87/13).

\section{Stimuli and apparatus}

Conditioned stimuli (CS) consisted of two pictures of female actresses with a neutral facial expression taken from the NimStim Set of Facial Expressions (Tottenham et al., 2009). Pictures were adjusted for luminance and brightness, converted to gray-scale and presented using Presentation (Neurobehavioral Systems, Inc, Albany, CA). One of the actresses was randomly selected as threat cue for each participant (CS+) while the other served as safety signal (CS-). Pictures were shown on a gray background on a 17-inch monitor (resolution $=1,280 \times 1,024$ pixel) in a flickering mode at a frequency of $12 \mathrm{~Hz}$ in order to elicit ssVEPs. Face-specific areas are often targeted with relatively slow driving frequencies (Baldauf and Desimone, 2014), but face-specific processing has also been isolated from paradigms with faster frequencies (Campagnoli et al., 2019; Gruss et al., 2012; Wieser et al., 2014a; Wieser et al., 2014c).

The US consisted of the respective CS+ face displaying a fearful expression and a simultaneously presented $95 \mathrm{~dB}$ shrill female scream of the IADS (Bradley and Lang, 1999). Four generalization stimuli (GS) were created by morphing the two faces in $20 \%$ steps using a face-morphing software (Squirlz Morph; Xiberpix, Solihull, UK). The GS most similar to the CS+ is referred to as GS1 and the GS most similar to the CS- as GS4 (see Figure 2).

\section{Design and procedure}

The experiment comprised three blocks (habituation, acquisition, generalization). Habituation and acquisition consisted of 30 trials (two faces, each presented 15 times), while there were 90 trials in the generalization phase (six faces, each presented 15 times), resulting in 150 trials in total. After completing the questionnaires, EEG electrodes were applied to participants, who were seated in a noise-reduced, darkened room one meter distant to the screen. In the habituation phase of the experiment, faces were presented for $3000 \mathrm{~ms}$ without reinforcement. During acquisition, one of the faces (CS+) was paired in 12 of 15 trials (80\% reinforcement) with the US, which lasted $1500 \mathrm{~ms}$ and was presented at the offset of the CS+ with a sound volume of $95 \mathrm{~dB}$ by Labtech speakers (Labtech International Ltd., Ringmer, East Sussex, GB) and a Kenwood KA-3010-Amplifier (Kenwood Electronics, Heusenstamm, GER). Subjects were not informed of any specific relation among the CSs and the US prior to the experiment and the assignment of faces to CS-conditions was counterbalanced 
across subjects. Generalization consisted of the CS+, CS-, and four GS, each presented 15 times (90 trials). While CS- and GS were never reinforced, 6 of the $15 \mathrm{CS}+$ were still followed by the US to prevent early extinction (40\% reinforcement, Lissek et al., 2008) (see Figure 2). The presentation order of the faces within each block was pseudo-randomized such that no more than two of the same faces could occur in a row. After each trial, a gray screen with a fixation cross was presented. Inter-trial intervals differed between 2000 and $2500 \mathrm{~ms}$. At the end of each phase, subjects rated the valence (ranging from 1 - 'very pleasant' to 9 - 'very unpleasant') and arousal (ranging from 1 - 'very calm' to 9 - 'very arousing') of the faces using a computer-based version of the Self-Assessment Manikin Scale (Bradley and Lang, 1994). Moreover, subjects were asked to rate US expectancy after acquisition and generalization from $0 \%$ to $100 \%$ as a response to the question 'What is the likelihood that the currently presented face is followed by a scream?' to measure successful learning of the CS-US association.

\section{EEG recording and analysis}

Electrocortical activity was measured via 129 electrodes using an Electrical Geodesics (EGl, Eugene, OR) high-density EEG System referenced to $\mathrm{Cz}$, recorded with a sampling rate of $250 \mathrm{~Hz}$ and online bandpass filtered with 0.1 and $100 \mathrm{~Hz}$ and a $50 \mathrm{~Hz}$ notch filter. The threshold of impedances was kept below $50 \mathrm{k} \Omega$ as recommended for the Electrical Geodesics high-impedance amplifiers. Offline, EEG analyses were implemented using the software EMEGS (Electro Magnetic Encephalography) for Matlab (Peyk et al., 2011). First, epochs of $600 \mathrm{~ms}$ pre-stimulus and $3000 \mathrm{~ms}$ post-stimulus onset were extracted, and data were filtered with a low-pass filter of $40 \mathrm{~Hz}$. In a second step, artifact rejection was conducted according to the SCADS procedure (Junghöfer et al., 2000). This way, outlying channels could be identified and interpolated from the full channel set and artifact-contaminated trials could be excluded from the analyses. Trials were rejected when more than 20 channels out of 129 were outliers as per the statistical parameters used for artifact identification. In a next step, remaining trials were averaged in the time domain for each subject according to the different experimental conditions. To increase spatial resolution of the EEG signal, we then calculated the current source densities (CSD) of the time-averaged data. The CSD transformation offers the advantage of reducing the negative impact of volume conduction and thereby effectively minimizing unwanted topographical variability between subjects by quantifying source densities (Junghöfer et al., 1997; Kayser and Tenke, 2015). The CSD approach relies on the spatial Laplacian (the second spatial derivative) of the scalp potential to estimate the potential distribution at the cortical surface. Here, we used the CSD algorithm described by Junghöfer et al., 1997, with $\lambda=0.2$, which is well suited for dense-array EEG montages and has been used in previous studies investigating ssVEP responses to facial stimuli (McTeague et al., 2011) or during fear generalization (McTeague et al., 2015). The CSD time series values were then transformed into the frequency domain using a Fast-Fourier-Transformation on a time interval between 500 and 3000 ms after stimulus-onset. The first 500 ms after stimulus onset were omitted due to initial non-stationary components of the ssVEP. In a next step, we obtained the signal-to-noise ratio (SNR) for the driving frequency of $12 \mathrm{~Hz}$ by dividing the power of the driving frequency by the mean of the spectral power at six adjacent frequency bins, leaving out the two immediate neighbors. The SNR is a unitless measure that accounts for both the evoked signal and the random noise in the data and has recently been used in other ssVEP paradigms as well (Barry-Anwar et al., 2018; Boylan et al., 2019).

For statistical analysis, the ssVEP activity was pooled across sensor $\mathrm{Oz}$ and seven neighbouring electrodes (EGI sensors 70, 71, 72, 74, 75, 76, 82, 83; Wieser et al., 2014a; Wieser and Keil, 2014b).

\section{Statistical analysis}

Stimulus differences in SsVEP amplitudes as well as valence and arousal ratings during habituation and acquisition were analyzed with linear mixed models with the within-subject factor CS-type (2: CS ,+ CS-) and mean-centered SPAl scores as covariates. Both main effects and the interaction of CStype and SPAl were entered as fixed effects. Subjects were entered as random intercepts to the model. For the generalization phase, the same linear mixed model was analyzed, though the factor CS-type now included six levels (CS+, GS1, GS2, GS3, GS4, CS-). Follow-up tests for significant effects of CS-type were analyzed using simple contrasts with the CS- as reference level (Lissek et al., 
2008). US expectancy ratings underwent the same analyses except for the habituation phase, because US expectancy ratings just started after the acquisition phase. Significance was evaluated using the Kenward-Rogers approximation for degrees of freedom (Kenward and Roger, 1997; Luke, 2017). Alpha was set at $p<0.05$ (two-tailed). Linear mixed models were conducted in the $R$ software environment (version 3.5.0; $R$ Development Core Team, 2020), using the package 'Ime4' (version 1.1-20; Bates et al., 2015). Standardized effect sizes and confidence intervals for the discrete factors of the linear mixed models were calculated as partial- $R^{2}$, using the package ' $r 2 \mathrm{glmm}$ ' (version 0.1.2; Jaeger et al., 2017).

To compare the lateral inhibition pattern to a quadratic and linear fit, Bayesian linear models were used. For this analysis, a pre-specified weight vector for each contrast entered the linear regression as predictors. The lateral inhibition pattern was expressed as the difference of two Gaussians (weights: $+2,-2,+0.5,+1,+0.5,-2$ for CS+, GS1, GS2, GS3, GS4, CS-). Note, that in contrast to the study of McTeague et al., 2015, generalization learning occurred along a continuum from CS+ to CS-, which is why the weight vector was adapted to only one half of the previously used model. For the quadratic and linear trends, the following weights were used, respectively: quadratic (weights: $+2.5334,+1.0934,-0.0267,-0.8267,-1.3067,-1.4667$ ) and linear (weights: $+2.5,+1.5$, $+0.5,-0.5,-1.5,-2.5$ ), paralleling previous work on fear generalization (Ahrens et al., 2016; Lissek et al., 2014a; Lissek et al., 2014b). The linear model analysis is mathematically insensitive to any linear transformation of the contrast vectors, which is why every vector was centered around zero to facilitate model comparisons. In each model, centered SPAI scores were entered as an additional predictor variable and subjects were entered as random intercepts to the model. Transitive Bayes factors (BFs) were then calculated for each candidate main effect, interaction and predictor weight model. Interpretation of Bayes factors follows guidelines developed by Jeffreys (1961). Bayesian analyses were conducted in R, using the package 'BayesFactor' (version 0.9) and default JZS-priors (Rouder et al., 2012). To follow up on the hierarchical model selection, individual visuocortical tuning indices were calculated for the lateral inhibition pattern. This index was defined as the scalar product of the weights of the lateral inhibition pattern and the subjects' individual ssVEPresponse to the corresponding stimulus, so that higher/lower visuocortical tuning indices indicate stronger/weaker accentuation of the lateral inhibition pattern. Similar indices were calculated for the quadratic and linear trend. In a next step, these indices were correlated with the SPAl scores. For the frequentist analysis, Pearson's $r$ was calculated, and the alpha level was set to .05, while the population correlation parameter $\rho$ was estimated for a two-sided Bayes factor analysis (Ly et al., 2016).

\section{Acknowledgements}

This work was supported by the German Research Foundation - project number 44541416 - TRR-58, projects B05 of the 2nd funding period and B01 of the 3rd funding period

\section{Additional information}

Funding

\begin{tabular}{lll} 
Funder & Grant reference number & Author \\
\hline $\begin{array}{l}\text { Deutsche Forschungsge- } \\
\text { meinschaft }\end{array}$ & $44541416-$ TRR-58 & Paul Pauli \\
& & Matthias J Wieser
\end{tabular}

The funders had no role in study design, data collection and interpretation, or the decision to submit the work for publication.

\section{Author contributions}

Yannik Stegmann, Data curation, Software, Formal analysis, Visualization, Writing - original draft, Writing - review and editing; Lea Ahrens, Conceptualization, Data curation, Investigation, Methodology; Paul Pauli, Conceptualization, Supervision, Funding acquisition, Visualization, Methodology, Project administration, Writing - review and editing; Andreas Keil, Data curation, Software, Supervision, Visualization, Methodology, Writing - review and editing; Matthias J Wieser, Conceptualization, 
Resources, Data curation, Software, Formal analysis, Supervision, Funding acquisition, Methodology, Project administration, Writing - review and editing

Author ORCIDs

Yannik Stegmann (iD) https://orcid.org/0000-0002-0933-8492

Ethics

Human subjects: Prior to participation, written informed consent was obtained from each participant.

The study was approved by the ethics review board of the University of Würzburg (87/13).

Decision letter and Author response

Decision letter https://doi.org/10.7554/eLife.55204.sa1

Author response https://doi.org/10.7554/eLife.55204.sa2

\section{Additional files}

Supplementary files

- Transparent reporting form

Data availability

Data sets, the code for all analyses, as well as the code for the production of Tables 1-4, Figure 3, Figure 5 and Figure 7, are available at https://osf.io/4965f/.

The following dataset was generated:

\begin{tabular}{|c|c|c|c|c|}
\hline Author(s) & Year & Dataset title & Dataset URL & $\begin{array}{l}\text { Database and } \\
\text { Identifier }\end{array}$ \\
\hline $\begin{array}{l}\text { Stegmann Y, Ah- } \\
\text { rens L, Pauli P, Keil } \\
\text { A, Wieser MJ }\end{array}$ & 2020 & $\begin{array}{l}\text { Social aversive generalization } \\
\text { learning sharpens the tuning of } \\
\text { visuocortical neurons to facial } \\
\text { identity cues }\end{array}$ & https://osf.io/4965f/ & $\begin{array}{l}\text { Open Science } \\
\text { Framework, } 4965 f\end{array}$ \\
\hline
\end{tabular}

\section{References}

Ahissar M, Hochstein S. 2004. The reverse hierarchy theory of visual perceptual learning. Trends in Cognitive Sciences 8:457-464. DOI: https://doi.org/10.1016/j.tics.2004.08.011

Ahrens LM, Pauli P, Reif A, Mühlberger A, Langs G, Aalderink T, Wieser MJ. 2016. Fear conditioning and stimulus generalization in patients with social anxiety disorder. Journal of Anxiety Disorders 44:36-46. DOI: https://doi.org/10.1016/j.janxdis.2016.10.003, PMID: 27728838

Antov MI, Plog E, Bierwirth P, Keil A, Stockhorst U. 2020. Visuocortical tuning to a threat-related feature persists after extinction and consolidation of conditioned fear. Scientific Reports 10:3926. DOI: https://doi.org/10.1038/ s41598-020-60597-z, PMID: 32127551

Baldauf D, Desimone R. 2014. Neural mechanisms of object-based attention. Science 344:424-427. DOI: https:// doi.org/10.1126/science.1247003, PMID: 24763592

Bantin T, Stevens S, Gerlach AL, Hermann C. 2016. What does the facial dot-probe task tell Us about attentional processes in social anxiety? A systematic review. Journal of Behavior Therapy and Experimental Psychiatry 50: 40-51. DOI: https://doi.org/10.1016/j.jbtep.2015.04.009, PMID: 26042381

Bar-Haim Y, Lamy D, Pergamin L, Bakermans-Kranenburg MJ, van IJzendoorn MH. 2007. Threat-related attentional Bias in anxious and nonanxious individuals: a meta-analytic study. Psychological Bulletin 133:1-24. DOI: https://doi.org/10.1037/0033-2909.133.1.1, PMID: 17201568

Barry-Anwar R, Hadley H, Conte S, Keil A, Scott LS. 2018. The developmental time course and topographic distribution of individual-level monkey face discrimination in the infant brain. Neuropsychologia 108:25-31. DOl: https://doi.org/10.1016/j.neuropsychologia.2017.11.019

Bates D, Mächler M, Bolker B, Walker S. 2015. Fitting linear Mixed-Effects models using Lme4. Journal of Statistical Software 67:48. DOI: https://doi.org/10.18637/jss.v067.i01

Beidel DC, Turner SM, Stanley MA, Dancu CV. 1989. The social phobia and anxiety inventory: concurrent and external validity. Behavior Therapy 20:417-427. DOI: https://doi.org/10.1016/S0005-7894(89)80060-7

Boecker L, Pauli P. 2019. Affective startle modulation and psychopathology: implications for appetitive and defensive brain systems. Neuroscience \& Biobehavioral Reviews 103:230-266. DOI: https://doi.org/10.1016/j. neubiorev.2019.05.019, PMID: 31129237 
Boylan MR, Kelly MN, Thigpen NN, Keil A. 2019. Attention to a threat-related feature does not interfere with concurrent attentive feature selection. Psychophysiology 56:e13332. DOI: https://doi.org/10.1111/psyp.13332, PMID: 30663061

Bradlley MM, Keil A, Lang PJ. 2012. Orienting and emotional perception: facilitation, attenuation, and interference. Frontiers in Psychology 3:493. DOI: https://doi.org/10.3389/fpsyg.2012.00493, PMID: 23181039

Bradley MM, Lang PJ. 1994. Measuring emotion: the Self-Assessment manikin and the semantic differential. Journal of Behavior Therapy and Experimental Psychiatry 25:49-59. DOl: https://doi.org/10.1016/0005-7916 (94)90063-9, PMID: 7962581

Bradley MM, Lang PJ. 1999. The International Affective Digitized Sounds (IADS): Stimuli Instruction Manual and Affective Ratings. NIMH Center for the Study of Emotion and Attention.

Bradley MM, Lang PJ. 2000. Emotion and Motivation. In: Cacioppo JT, Tassinary LG, Berntson G (Eds). Handbook of Psychophysiology. Cambridge University Press. p. 602-642.

Campagnoli RR, Wieser MJ, Gruss LF, Boylan MR, McTeague LM, Keil A. 2019. How the visual brain detects emotional changes in facial expressions: evidence from driven and intrinsic brain oscillations. Cortex 111:35-50. DOI: https://doi.org/10.1016/j.cortex.2018.10.006, PMID: 30447483

Chang L, Tsao DY. 2017. The code for facial identity in the primate brain. Cell 169:1013-1028. DOI: https://doi. org/10.1016/j.cell.2017.05.011, PMID: 28575666

Clark DM, Wells A. 1995. A cognitive model of social phobia. In: Heimberg RG (Ed). Social Phobia: Diagnosis, Assessment, and Treatment. New York: Guilford Press. p. 69-93.

Davis M. 2006. Neural systems involved in fear and anxiety measured with fear-potentiated startle. American Psychologist 61:741-756. DOI: https://doi.org/10.1037/0003-066X.61.8.741, PMID: 17115806

Dragoi V, Sharma J, Sur M. 2000. Adaptation-induced plasticity of orientation tuning in adult visual cortex. Neuron 28:287-298. DOI: https://doi.org/10.1016/S0896-6273(00)00103-3, PMID: 11087001

Duchaine B, Yovel G. 2015. A revised neural framework for face processing. Annual Review of Vision Science 1: 393-416. DOI: https://doi.org/10.1146/annurev-vision-082114-035518, PMID: 28532371

Dymond S, Dunsmoor JE, Vervliet B, Roche B, Hermans D. 2015. Fear generalization in humans: systematic review and implications for anxiety disorder research. Behavior Therapy 46:561-582. DOI: https://doi.org/10. 1016/j.beth.2014.10.001, PMID: 26459838

Efron B. 2012. Bayesian inference and the parametric bootstrap. The Annals of Applied Statistics 6:1971-1997. DOI: https://doi.org/10.1214/12-AOAS571, PMID: 23843930

Freiwald WA, Tsao DY, Livingstone MS. 2009. A face feature space in the macaque temporal lobe. Nature Neuroscience 12:1187-1196. DOI: https://doi.org/10.1038/nn.2363, PMID: 19668199

Freiwald WA, Tsao DY. 2010. Functional compartmentalization and viewpoint generalization within the macaque face-processing system. Science 330:845-851. DOI: https://doi.org/10.1126/science.1194908, PMID: 21051642

Fullana MA, Harrison BJ, Soriano-Mas C, Vervliet B, Cardoner N, Àvila-Parcet A, Radua J. 2016. Neural signatures of human fear conditioning: an updated and extended meta-analysis of $\mathrm{fMRI}$ studies. Molecular Psychiatry 21:500-508. DOI: https://doi.org/10.1038/mp.2015.88, PMID: 26122585

Furr RM, Rosenthal R. 2003. Repeated-measures contrasts for "multiple-pattern" hypotheses. Psychological Methods 8:275-293. DOI: https://doi.org/10.1037/1082-989X.8.3.275, PMID: 14596491

Gilaie-Dotan S, Malach R. 2007. Sub-exemplar shape tuning in human face-related Areas. Cerebral Cortex 17: 325-338. DOI: https://doi.org/10.1093/cercor/bhj150, PMID: 16525131

Gruss LF, Wieser MJ, Schweinberger SR, Keil A. 2012. Face-evoked steady-state visual potentials: effects of presentation rate and face inversion. Frontiers in Human Neuroscience 6:16. DOI: https://doi.org/10.3389/ fnhum.2012.00316, PMID: 23205009

Jaeger BC, Edwards LJ, Das K, Sen PK. 2017. An R2 statistic for fixed effects in the generalized linear mixed model. Journal of Applied Statistics 44:1086-1105. DOI: https://doi.org/10.1080/02664763.2016.1193725

Junghöfer M, Elbert T, Leiderer P, Berg P, Rockstroh B. 1997. Mapping EEG-potentials on the surface of the brain: a strategy for uncovering cortical sources. Brain Topography 9:203-217. DOI: https://doi.org/10.1007/ BF01190389, PMID: 9104831

Junghöfer M, Elbert T, Tucker DM, Rockstroh B. 2000. Statistical control of artifacts in dense array EEG/MEG studies. Psychophysiology 37:523-532. DOI: https://doi.org/10.1111/1469-8986.3740523, PMID: 10934911

Kaczkurkin AN, Burton PC, Chazin SM, Manbeck AB, Espensen-Sturges T, Cooper SE, Sponheim SR, Lissek S. 2017. Neural substrates of overgeneralized conditioned fear in PTSD. American Journal of Psychiatry 174:125134. DOI: https://doi.org/10.1176/appi.ajp.2016.15121549, PMID: 27794690

Kayser J, Tenke CE. 2015. On the benefits of using surface laplacian (current source density) methodology in electrophysiology. International Journal of Psychophysiology 97:171-173. DOI: https://doi.org/10.1016/j. ijpsycho.2015.06.001, PMID: 26071227

Keil A, Gruber T, Müller MM, Moratti S, Stolarova M, Bradley MM, Lang PJ. 2003. Early modulation of visual perception by emotional arousal: evidence from steady-state visual evoked brain potentials. Cognitive, Affective, \& Behavioral Neuroscience 3:195-206. DOI: https://doi.org/10.3758/CABN.3.3.195, PMID: 14672156

Keil A, Miskovic V, Gray MJ, Martinovic J. 2013. Luminance, but not chromatic visual pathways, mediate amplification of conditioned danger signals in human visual cortex. European Journal of Neuroscience 38: 3356-3362. DOI: https://doi.org/10.1111/ejn.12316

Kemp AH, Gray MA, Eide P, Silberstein RB, Nathan PJ. 2002. Steady-state visually evoked potential topography during processing of emotional valence in healthy subjects. Neurolmage 17:1684-1692. DOI: https://doi.org/ 10.1006/nimg.2002.1298, PMID: 12498742 
Kenward MG, Roger JH. 1997. Small sample inference for fixed effects from restricted maximum likelihood. Biometrics 53:983-997. DOI: https://doi.org/10.2307/2533558, PMID: 9333350

LeDoux JE, Pine DS. 2016. Using neuroscience to help understand fear and anxiety: a Two-System framework. American Journal of Psychiatry 173:1083-1093. DOI: https://doi.org/10.1176/appi.ajp.2016.16030353, PMID: 27609244

Leopold DA, Bondar IV, Giese MA. 2006. Norm-based face encoding by single neurons in the monkey inferotemporal cortex. Nature 442:572-575. DOI: https://doi.org/10.1038/nature04951, PMID: 16862123

Lissek S, Biggs AL, Rabin SJ, Cornwell BR, Alvarez RP, Pine DS, Grillon C. 2008. Generalization of conditioned fear-potentiated startle in humans: experimental validation and clinical relevance. Behaviour Research and Therapy 46:678-687. DOI: https://doi.org/10.1016/j.brat.2008.02.005, PMID: 18394587

Lissek S, Rabin S, Heller RE, Lukenbaugh D, Geraci M, Pine DS, Grillon C. 2010. Overgeneralization of conditioned fear as a pathogenic marker of panic disorder. American Journal of Psychiatry 167:47-55. DOI: https://doi.org/10.1176/appi.ajp.2009.09030410, PMID: 19917595

Lissek S, Bradford DE, Alvarez RP, Burton P, Espensen-Sturges T, Reynolds RC, Grillon C. 2014a. Neural substrates of classically conditioned fear-generalization in humans: a parametric fMRI study. Social Cognitive and Affective Neuroscience 9:1134-1142. DOI: https://doi.org/10.1093/scan/nst096, PMID: 23748500

Lissek S, Kaczkurkin AN, Rabin S, Geraci M, Pine DS, Grillon C. 2014b. Generalized anxiety disorder is associated with overgeneralization of classically conditioned fear. Biological Psychiatry 75:909-915. DOI: https://doi.org/ 10.1016/j.biopsych.2013.07.025, PMID: 24001473

Loffler G, Yourganov G, Wilkinson F, Wilson HR. 2005. fMRI evidence for the neural representation of faces. Nature Neuroscience 8:1386-1391. DOI: https://doi.org/10.1038/nn1538, PMID: 16136037

Luke SG. 2017. Evaluating significance in linear mixed-effects models in R. Behavior Research Methods 49:14941502. DOI: https://doi.org/10.3758/s13428-016-0809-y, PMID: 27620283

Ly A, Verhagen J, Wagenmakers E-J. 2016. Harold Jeffreys's default Bayes factor hypothesis tests: Explanation, extension, and application in psychology. Journal of Mathematical Psychology 72:19-32. DOI: https://doi.org/ 10.1016/j.jmp.2015.06.004

McTeague LM, Shumen JR, Wieser MJ, Lang PJ, Keil A. 2011. Social vision: sustained perceptual enhancement of affective facial cues in social anxiety. Neurolmage 54:1615-1624. DOI: https://doi.org/10.1016/j. neuroimage.2010.08.080, PMID: 20832490

McTeague LM, Gruss LF, Keil A. 2015. Aversive learning shapes neuronal orientation tuning in human visual cortex. Nature Communications 6:7823. DOI: https://doi.org/10.1038/ncomms8823, PMID: 26215466

McTeague LM, Laplante MC, Bulls HW, Shumen JR, Lang PJ, Keil A. 2018. Face perception in social anxiety: visuocortical dynamics reveal propensities for hypervigilance or avoidance. Biological Psychiatry 83:618-628. DOI: https://doi.org/10.1016/j.biopsych.2017.10.004, PMID: 29157845

Miskovic V, Keil A. 2012. Acquired fears reflected in cortical sensory processing: a review of electrophysiological studies of human classical conditioning. Psychophysiology 49:1230-1241. DOl: https://doi.org/10.1111/j.14698986.2012.01398.x, PMID: 22891639

Miskovic V, Keil A. 2013. Visuocortical changes during delay and trace aversive conditioning: evidence from steady-state visual evoked potentials. Emotion 13:554-561. DOI: https://doi.org/10.1037/a0031323, PMID: 233 98582

Moratti S, Keil A. 2005. Cortical activation during pavlovian fear conditioning depends on heart rate response patterns: an MEG study. Cognitive Brain Research 25:459-471. DOI: https://doi.org/10.1016/j.cogbrainres. 2005.07.006, PMID: 16140512

Moratti S, Keil A. 2009. Not what you expect: experience but not expectancy predicts conditioned responses in human visual and supplementary cortex. Cerebral Cortex 19:2803-2809. DOI: https://doi.org/10.1093/cercor/ bhp052, PMID: 19304914

Mühlberger A, Wieser MJ, Herrmann MJ, Weyers P, Tröger C, Pauli P. 2009. Early cortical processing of natural and artificial emotional faces differs between lower and higher socially anxious persons. Journal of Neural Transmission 116:735-746. DOl: https://doi.org/10.1007/s00702-008-0108-6, PMID: 18784899

Müller MM, Teder-Sälejärvi W, Hillyard SA. 1998. The time course of cortical facilitation during cued shifts of spatial attention. Nature Neuroscience 1:631-634. DOI: https://doi.org/10.1038/2865, PMID: 10196572

Müller MM, Andersen S, Trujillo NJ, Valdés-Sosa P, Malinowski P, Hillyard SA. 2006. Feature-selective attention enhances color signals in early visual Areas of the human brain. PNAS 103:14250-14254. DOI: https://doi.org/ 10.1073/pnas.0606668103, PMID: 16956975

Nelson BD, Weinberg A, Pawluk J, Gawlowska M, Proudfit GH. 2015. An Event-Related potential investigation of fear generalization and intolerance of uncertainty. Behavior Therapy 46:661-670. DOI: https://doi.org/10.1016/ j.beth.2014.09.010, PMID: 26459846

Onat S, Büchel C. 2015. The neuronal basis of fear generalization in humans. Nature Neuroscience 18:18111818. DOI: https://doi.org/10.1038/nn.4166, PMID: 26571459

Peyk P, De Cesarei A, Junghöfer M. 2011. ElectroMagnetoEncephalography software: overview and integration with other EEG/MEG toolboxes. Computational Intelligence and Neuroscience 2011:1-10. DOI: https://doi. org/10.1155/2011/861705, PMID: 21577273

R Development Core Team. 2020. R: A language and environment for statistical computing. Vienna, Austria: R Foundation for Statistical Computing http://www.r-project.org.

Rapee RM, Heimberg RG. 1997. A cognitive-behavioral model of anxiety in social phobia. Behaviour Research and Therapy 35:741-756. DOI: https://doi.org/10.1016/S0005-7967(97)00022-3, PMID: 9256517 
Robinson OJ, Vytal K, Cornwell BR, Grillon C. 2013. The impact of anxiety upon cognition: perspectives from human threat of shock studies. Frontiers in Human Neuroscience 7:203. DOI: https://doi.org/10.3389/fnhum. 2013.00203, PMID: 23730279

Rossion B. 2014. Understanding face perception by means of prosopagnosia and neuroimaging. Frontiers in Bioscience 6:258-307. DOI: https://doi.org/10.2741/e706

Rouder JN, Morey RD, Speckman PL, Province JM. 2012. Default bayes factors for ANOVA designs. Journal of Mathematical Psychology 56:356-374. DOI: https://doi.org/10.1016/j.jmp.2012.08.001

Sjouwerman R, Niehaus J, Kuhn M, Lonsdorf TB. 2016. Don't startle me-Interference of startle probe presentations and intermittent ratings with fear acquisition. Psychophysiology 53:1889-1899. DOI: https://doi. org/10.1111/psyp.12761, PMID: 27628268

Stegmann Y, Reicherts P, Andreatta M, Pauli P, Wieser MJ. 2019a. The effect of trait anxiety on attentional mechanisms in combined context and cue conditioning and extinction learning. Scientific Reports 9:8855. DOI: https://doi.org/10.1038/s41598-019-45239-3, PMID: 31222028

Stegmann Y, Schiele MA, Schümann D, Lonsdorf TB, Zwanzger P, Romanos M, Reif A, Domschke K, Deckert J, Gamer M, Pauli P. 2019b. Individual differences in human fear generalization-pattern identification and implications for anxiety disorders. Translational Psychiatry 9:307. DOI: https://doi.org/10.1038/s41398-0190646-8, PMID: 31740663

Struyf D, Zaman J, Hermans D, Vervliet B. 2017. Gradients of fear: how perception influences fear generalization. Behaviour Research and Therapy 93:116-122. DOI: https://doi.org/10.1016/j.brat.2017.04.001, PMID: 2 8410461

Tinoco-González D, Fullana MA, Torrents-Rodas D, Bonillo A, Vervliet B, Blasco MJ, Farré M, Torrubia R. 2015. Conditioned fear acquisition and generalization in generalized anxiety disorder. Behavior Therapy 46:627-639. DOI: https://doi.org/10.1016/j.beth.2014.12.004, PMID: 26459843

Torrents-Rodas D, Fullana MA, Bonillo A, Caseras X, Andión O, Torrubia R. 2013. No effect of trait anxiety on differential fear conditioning or fear generalization. Biological Psychology 92:185-190. DOI: https://doi.org/10. 1016/j.biopsycho.2012.10.006, PMID: 23131617

Tottenham N, Tanaka JW, Leon AC, McCarry T, Nurse M, Hare TA, Marcus DJ, Westerlund A, Casey BJ, Nelson C. 2009. The NimStim set of facial expressions: judgments from untrained research participants. Psychiatry Research 168:242-249. DOI: https://doi.org/10.1016/j.psychres.2008.05.006, PMID: 19564050

Weike Al, Schupp HT, Hamm AO. 2007. Fear acquisition requires awareness in trace but not delay conditioning. Psychophysiology 44:170-180. DOI: https://doi.org/10.1111/j.1469-8986.2006.00469.x, PMID: 17241153

Wieser MJ, Pauli P, Mühlberger A. 2009. Probing the attentional control theory in social anxiety: an emotional saccade task. Cognitive, Affective, \& Behavioral Neuroscience 9:314-322. DOl: https://doi.org/10.3758/CABN. 9.3.314, PMID: 19679766

Wieser MJ, Pauli P, Reicherts P, Mühlberger A. 2010. Don't look at me in anger! enhanced processing of angry faces in anticipation of public speaking. Psychophysiology 47:271-280. DOl: https://doi.org/10.1111/j.14698986.2009.00938.x, PMID: 20030758

Wieser MJ, Flaisch T, Pauli P. 2014a. Raised middle-finger: electrocortical correlates of social conditioning with nonverbal affective gestures. PLOS ONE 9:e102937. DOI: https://doi.org/10.1371/journal.pone.0102937, PMID: 25054341

Wieser MJ, Miskovic V, Rausch S, Keil A. 2014c. Different time course of visuocortical signal changes to fearconditioned faces with direct or averted gaze: a ssVEP study with single-trial analysis. Neuropsychologia $\mathbf{6 2}$ : 101-110. DOI: https://doi.org/10.1016/j.neuropsychologia.2014.07.009, PMID: 25050854

Wieser MJ, Miskovic V, Keil A. 2016. Steady-state visual evoked potentials as a research tool in social affective neuroscience. Psychophysiology 53:1763-1775. DOI: https://doi.org/10.1111/psyp.12768, PMID: 27699794

Wieser MJ, Keil A. 2014b. Fearful faces heighten the cortical representation of contextual threat. Neurolmage 86:317-325. DOI: https://doi.org/10.1016/j.neuroimage.2013.10.008, PMID: 24125792

Yuan M, Giménez-Fernández T, Méndez-Bértolo C, Moratti S. 2018. Ultrafast cortical gain adaptation in the human brain by Trial-To-Trial changes of associative strength in fear learning. The Journal of Neuroscience 38 : 8262-8276. DOI: https://doi.org/10.1523/JNEUROSCI.0977-18.2018, PMID: 30104342

Zaman J, Ceulemans E, Hermans D, Beckers T. 2019. Direct and indirect effects of perception on generalization gradients. Behaviour Research and Therapy 114:44-50. DOI: https://doi.org/10.1016/j.brat.2019.01.006, PMID: 30771704 